\title{
Evaluación sensorial del vino artesanal de uva Isabella (Vitis labrusca L.)
}

\section{Sensory evaluation of the wine grape Isabella (Vitis labrusca L.) hand crafted}

Fecha recepción: 25 de febrero de 2015

Fecha aprobación: 2 de mayo de 2015

\author{
Pedro José Almanza-Merchán ${ }^{1}$, Andrea Johana Reyes-M² \\ Marco Luis Ayala ${ }^{3}$, William Balaguera-L ${ }^{4}$ \\ Pablo Antonio Serrano-Cely ${ }^{5}$
}

\section{Resumen}

El consumo de vino en Colombia se ha incrementado durante los últimos años, en especial el de los vinos tinto. A pesar de las pocas características vínicas de la uva Isabella (Vitis labrusca L.), su bajo contenido de azúcares y su alta concentración de ácidos le otorgan el potencial para la elaboración de un vino que presenta sabores y aromas intensos. La combinación de color, aroma y sabor provoca la denominada textura del vino. El análisis sensorial de los vinos, a través de catas, da origen a una escala entre los vinos y a una descripción que genera cierto impacto en el posicionamiento de este licor en el mercado. Esta investigación buscó evaluar la calidad organoléptica del vino artesanal de uva Isabella, mediante una cata a ciegas. Se evaluaron tres tipos de vino; dos producidos en la Universidad Pedagógica y Tecnológica de Colombia (semiseco y semidulce) y uno procedente del municipio de Cerrito, Valle del Cauca. El panel conformado por 20 catadores evaluó los vinos de acuerdo con los parámetros establecidos por el Concurso Internacional de Vinos y Espirituosos (CINVE). Los resultados presentaron diferencias significativas entre el vino Portal del Paraíso ${ }^{\circledR}$ y los vinos Uptc. Los vinos Uptc obtuvieron la menor puntuación, catalogándolos, de acuerdo con el CINVE, como los mejores sensorialmente.

Palabras clave: vino, cata de vinos, bouquet, calidad organoléptica, vino tinto.

\footnotetext{
Abstract

1 Ph.D. Universidad Pedagógica y Tecnológica de Colombia (Tunja-Boyacá, Colombia).pedro.almanza@uptc.edu.co.

2 Profesional Independiente (Tunja-Boyacá, Colombia).

3 Profesional Independiente (Tunja-Boyacá, Colombia).

4 Universidad Nacional de Colombia (Bogotá-Distrito Capital-Colombia).

5 M.Sc. Universidad Pedagógica y Tecnológica de Colombia (Tunja-Boyacá, Colombia).
} 
The production and consumption of wine in Colombia have increased over the last decade, especially with the red wines which are preferred by Colombians. Spite the few organoleptic characteristics of the grape Isabella (Vitis labrusca L.), it possesses intense flavors and aromas, plus its low sugar content and high concentration of acids, gives it the potential to be used in winemaking. The parameters involved in the sensory evaluation of the wines are the color, aroma and flavor and become sensations perceived in different areas of the tongue. The combination of these causes's sensations are called wine texture. Sensory analysis of wines through tastings gives rise to a description, that although subjective, generates some impact on the market position of this drink. Descriptive tests are the most common and can express the different sensations of wine labeling them by a scale. The aim of this study was to evaluate the organoleptic quality through blind tasting, the artisan wine grape Isabella (Vitis labrusca L). We valued three crafted wines, two of them produced in the Pedagogical and Technological University of Colombia, and a third from the town of Cerrito, Valle del Cauca. The panel conformed by 20 tasters evaluated the wines according to the parameters set by the profile sheet given by the International Competition of Wines and Spirits (CINVE). The results showed significant differences between the Paradise wine Portal and the Semi-dry and semi-sweet UPTC wines. The results suggest that the Semi-dry wine obtained the lowest score ranking, as the best sensorial level. The Uptc wines obtained the lowest score, classified as the best in the sensory study.

Keywords: wine tasting, bouquet, organoleptic quality, red wine. 


\section{Introducción}

La uva Isabella (Vitis labrusca L.) es una especie híbrida originaria del sur de los Estados Unidos (1); los principales productores y exportadores de ella son Brasil, Colombia, Uruguay, Perú, Argentina y Chile (2). En Colombia, el Valle del Cauca presenta la mayor superficie sembrada de uva Isabella, seguido de Huila, que es el mayor productor nacional, con un rendimiento de 9,98 t.ha ${ }^{-1}$ (3), y en tercer lugar el departamento de Santander (4). La uva Isabella se consume comúnmente como fruta fresca y en jaleas, y gracias a su alta concentración de ácidos se usa para elaborar vinos (5).

Las bebidas vínicas han venido posicionándose en la última década como unas de las más apetecidas en Colombia, debido a su contenido de antioxidantes, como el resveratrol, compuesto que se encuentra especialmente en el vino tinto (6).

Los parámetros involucrados en la evaluación sensorial de los vinos son el color, el aroma y el sabor. El color del vino es una de las principales características organolépticas que se utilizan para establecer su calidad y aceptabilidad; la medición de este parámetro durante los paneles sensoriales es compleja, laboriosa, costosa y sujeta a error, debido a la subjetividad del juicio (7). El color del vino es una percepción abstracta, definida por su intensidad y profundidad. Las condiciones de observación varían de acuerdo con la luminosidad del lugar en donde se realice la cata; si la luminosidad es baja, el color se tornará oscuro, con una mala tonalidad; por ello, es importante que el tono sea neutro, para apreciar los matices reales de la coloración (8).

Las percepciones olfativas, vía retronasal o directa, dan una idea más específica de los criterios importantes para juzgar la calidad del vino; se denomina aroma a las sensaciones de los vinos jóvenes, y bouquet, al olor adquirido por los vinos envejecidos. Al referirnos al aroma de vinos jóvenes, este no es más que la mezcla del aroma primario, conocido como varietal, y el aroma secundario, que es originado por la fermentación. El sabor del vino se cataloga como dulce, sabor que aparece instantáneamente al llevar el vino a la boca; el sabor salado se percibe paulatinamente y solo se mantiene por cierto tiempo; la sensación ácida aumenta durante el tiempo en el que se mantenga el vino en la boca, y se percibe amargo con cada toma de un trago de vino. Estas sensaciones son percibidas en diferentes zonas de la lengua. La combinación de estas sensaciones provoca la denominada textura del vino (9).

El análisis sensorial de los vinos consiste en la descripción de estos en función de las propiedades organolépticas que los caracterizan. Dicho análisis permite evaluar los distintos tipos de vino, así como apreciar ciertos matices de algunas características dentro de la cata (10). La evaluación sensorial de los vinos a través de sesiones de cata es el método universalmente aceptado para definir su calidad. El evaluador es un verdadero aparato de medida, y uno de ellos es una repetición. Pese a la inherente subjetividad del análisis, este puede tener determinado efecto en el posicionamiento y comercialización del vino.

Se ha coincidido en la necesidad de tres requisitos claves que determinan la validez interna de las catas: que estas sean ciegas, que los juicios de los catadores sean independientes y que los resultados sean analizados de forma sistemática (10). Sin embargo, existen diferentes tipos de paneles sensoriales con particularidades específicas: el panel de expertos, conformado por personas de gran experiencia, en muchos casos enólogos reconocidos; el panel de jueces entrenados, integrado por personas capacitadas para actuar como evaluadores capaces de percibir las sensaciones por su conocimiento y algún tipo de experiencia en la evaluación sensorial, y el panel de jueces evaluadores, consumidores de vino elegidos al azar (11).

Las pruebas descriptivas o de perfil sensorial son las más comunes y permiten describir las diferentes sensaciones de los vinos, calificándolos a través de una escala. La técnica descriptiva con consenso de los evaluadores es la más utilizada. Se pueden utilizar escalas ordenadas de acuerdo con el orden lógico de degustación: primero la vista, luego el olfato $y$, finalmente, el gusto; aunque hay quienes prefieren no estructurarlas de esta manera 
(11). Catania y Avagnina (11) describe que el éxito de este análisis está ligado al entrenamiento de los evaluadores, con referencias base que permitan desarrollar una terminología dentro del panel.

En los análisis descriptivos de vinos es frecuente utilizar escalas conocidas por los jueces y, con ello, presentar de forma muy sencilla la descripción sensorial del producto analizado (12). Estas representaciones permiten, además, comparar de forma visual las diferencias sensoriales entre vinos. Se sugiere que los datos obtenidos durante la evaluación pueden ser analizados mediante análisis de varianza (ANOVA), interpretando las percepciones de los jueces frente a los vinos (11). Con base en lo anterior, el objetivo de este trabajo fue evaluar la calidad organoléptica, mediante la cata a ciegas, de vino artesanal de uva Isabella (Vitis labrusca L).

\section{Materiales y métodos}

Se realizó el análisis sensorial a ciegas a tres vinos elaborados artesanalmente; dos de ellos elaborados en la Universidad Pedagógica y Tecnológica de Colombia, y el tercero (testigo), en el municipio del Cerrito, Valle del Cauca, denominado Portal del Paraíso ${ }^{\circ}$.

La evaluación sensorial se desarrolla entre las 2:00 y 4:00 de la tarde, en el laboratorio de Fisiología Vegetal de la Universidad Pedagógica y Tecnológica de Colombia, el cual fue adecuado para esta actividad según las normas establecidas por la OIV; el panel estuvo conformado por 20 catadores (docentes y estudiantes del Programa de Ingeniería Agronómica), 14 de los cuales fueron entrenados en el análisis descriptivo y valorativo del vino de uva Isabella, y 6 no tenían ninguna experiencia. Para el desarrollo de la sesión de la cata a ciegas fue utilizada la ficha propuesta para el Concurso Internacional de Vinos y
Espirituosos (CINVE) (13), en la cual la menor valoración corresponde al mejor vino; así, los vinos catalogados como excelente están entre 0 y 10; los muy bien, entre 11 y 30; los bien, entre 31 y 50; los correcto, entre 51 y 70; los regular, entre 71 y 90, y los defectuoso, entre 91 y 100. Para el análisis cualitativo de la ficha se realizó una representación gráfica de la percepción sensorial de los tres vinos. Para determinar si existieron diferencias significativas en la valoración de los vinos se realizó un análisis de la parte cuantitativa de la ficha de cata, con un ANAVA y una prueba comparativa de Tukey, mediante la utilización del software SAS v. 8.1e (Cary, N.C).

\section{Resultados y discusión}

\section{Color}

De acuerdo con la ficha de la cata, el vino Portal del Paraíso ${ }^{\circ}$ sobresale por su profundidad oscura, con el $80 \%$, contrario a los vinos Semiseco y Semidulce, que tienen un valor medio, con el $65 \%$ y el $45 \%$, respectivamente (Fig. $1 \mathrm{~A}$ ). El $55 \%$ de los evaluadores describieron el vino Semiseco con una tonalidad rubí, el $40 \%$ describieron el Semidulce con el color rojo-púrpura, y el $70 \%$ describieron el Portal del Paraíso ${ }^{\circledR}$ con el color marrón (Fig. 1B), coincidiendo con reportes sobre la presencia de las mismas tonalidades en vinos tintos jóvenes de la denominación de origen "Rioja" (14); adicional a esto, Portal del Paraíso ${ }^{\oplus}$ fue descrito con el color ladrillo por el 25\%; estas tonalidades del Portal del Paraíso corresponden a vinos viejos (8) o, posiblemente, a la degradación del color por altas temperaturas durante la fermentación alcohólica, los cuales podría dar una sensación de profundidad oscura, como ocurrió en este caso. Se pudo apreciar la diferencia en la intensidad del color en cuanto a su limpidez de los vinos Semiseco y Semidulce frente al vino Portal del Paraíso . 
(A)
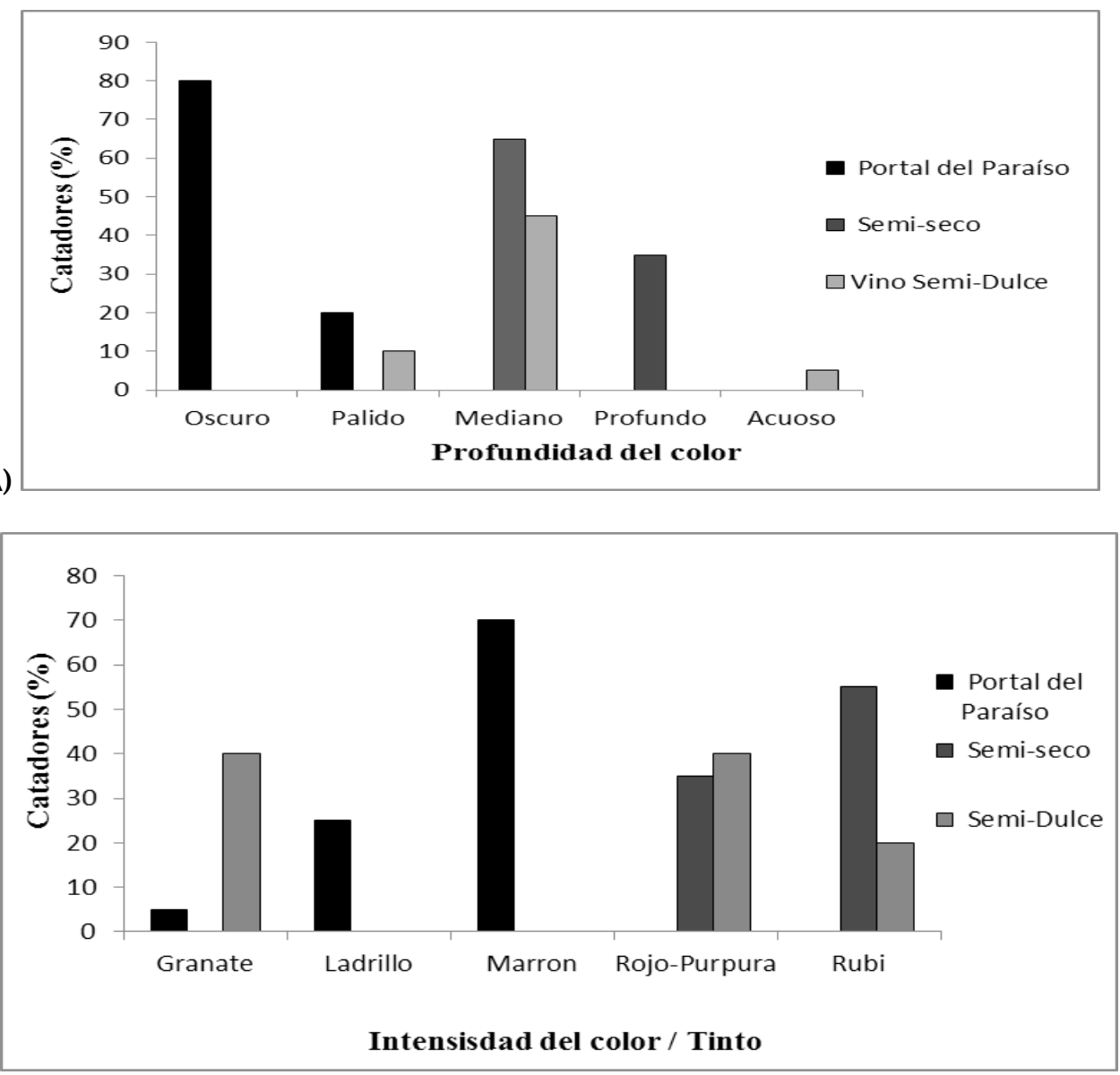

(B)

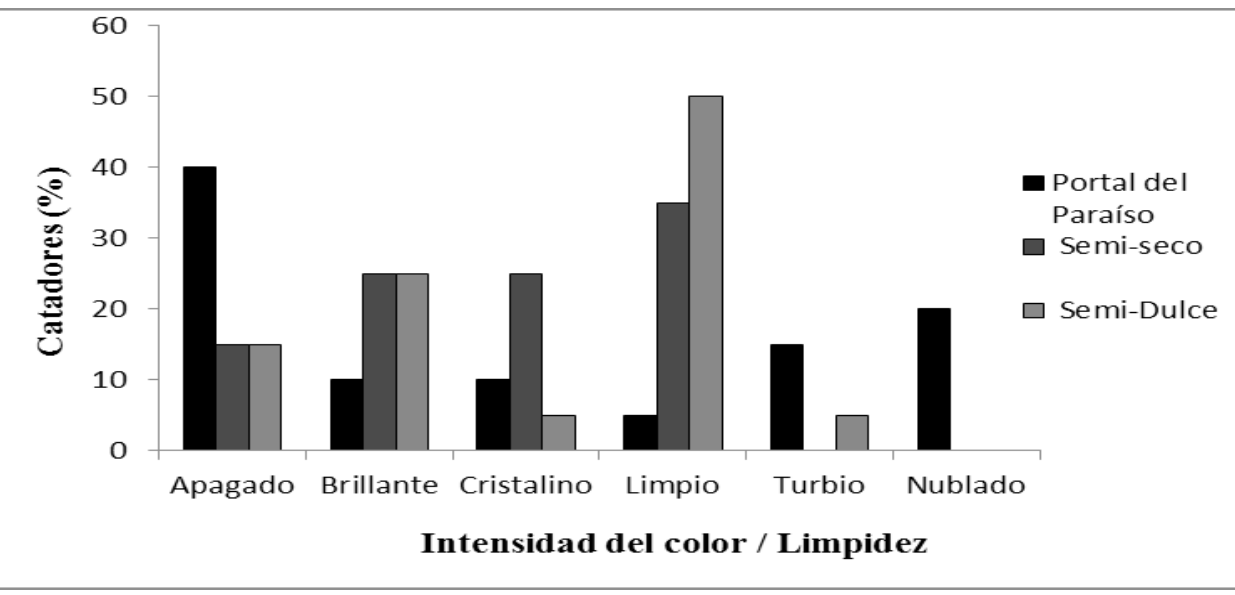

Figura 1. Percepción sensorial de vinos artesanales de uva Isabella A: Profundidad del color, B: intensidad del color/tinto y C: intensidad del color/limpidez de los vinos. 
La limpidez del vino Portal del Paraíso ${ }^{\oplus}$ correspondió a apagada, de acuerdo con el $40 \%$ de los evaluadores, y la de los vinos Semiseco y Semidulce, a limpios, con el $35 \%$ y el $50 \%$ en cada caso; en estos últimos también se presentaron similitudes en su brillantez, con el 25\% para los dos vinos, características de un buen vino (15) (Fig. 1C); estos autores (15) describen que, por el contrario, un vino turbio es señal de que sufrió problemas en su elaboración; por tanto, la limpidez es un factor altamente correlacionado con la coloración del vino.

\section{Aroma}

De acuerdo con los evaluadores, los tres vinos se consideraron aromáticos, con valores del $45 \%$, para Portal del Paraíso ${ }^{\oplus}$, y $35 \%$ para los vinos Semiseco y Semidulce (Fig. 2). El aroma expresado por los catadores correspondió al frutal en los tres casos: Portal del Paraíso obtuvo el $45 \%$; el Semiseco, el $60 \%$, y el Semidulce, el $50 \%$. Para el vino Portal de Paraíso ${ }^{\oplus}$, algunos catadores tuvieron una apreciación un poco más específica, mencionando sensaciones como aromas de panela, madera y miel, todos con el 5\% (Fig. 3). En (16) se describen características similares en vinos de la variedad Cinsaut, que tienen aromas frutales de intensidad media. Los ésteres etílicos de los ácidos grasos y las $\gamma$-lactonas alifáticas contribuyen de manera neta en la nota aromática, así como en la sensación frutal del aroma del vino (17). De la misma forma, la percepción aromática sería el resultado de la interacción de estos componentes con otras sustancias monoterpenoicas, compartiendo alguna similitud aromática, lo que concuerda con los resultados obtenidos.

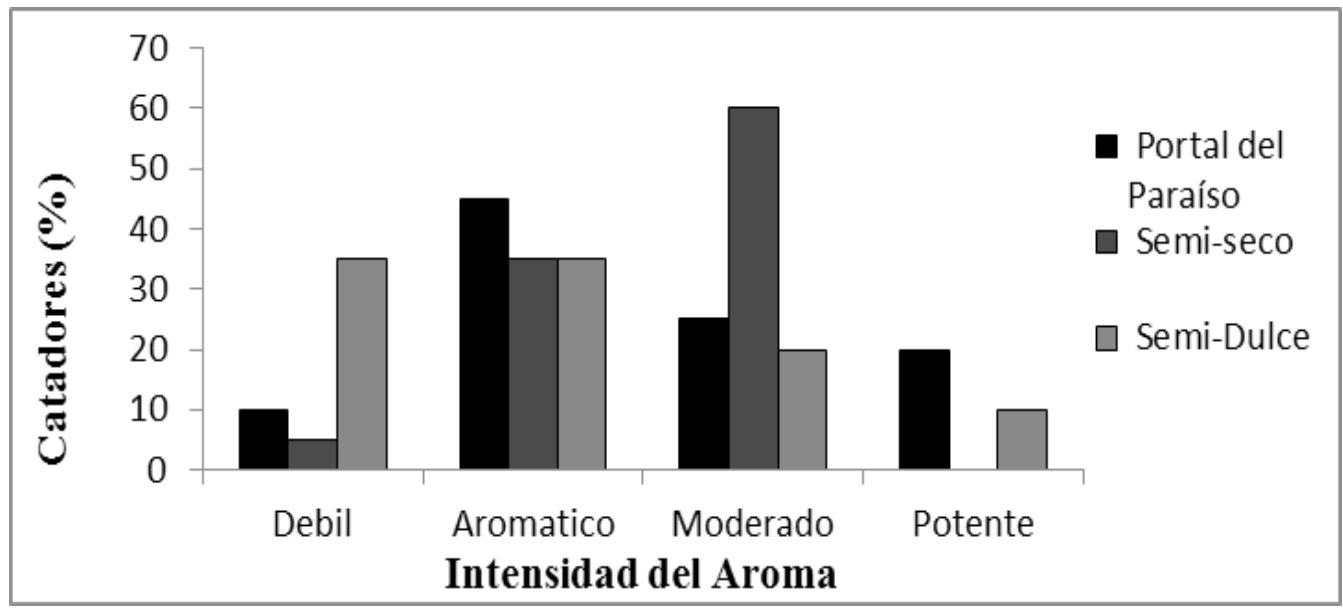

Figura 2. Percepción sensorial de la intensidad del aroma de los vinos Portal del Paraíso, Semiseco y Semidulce. 


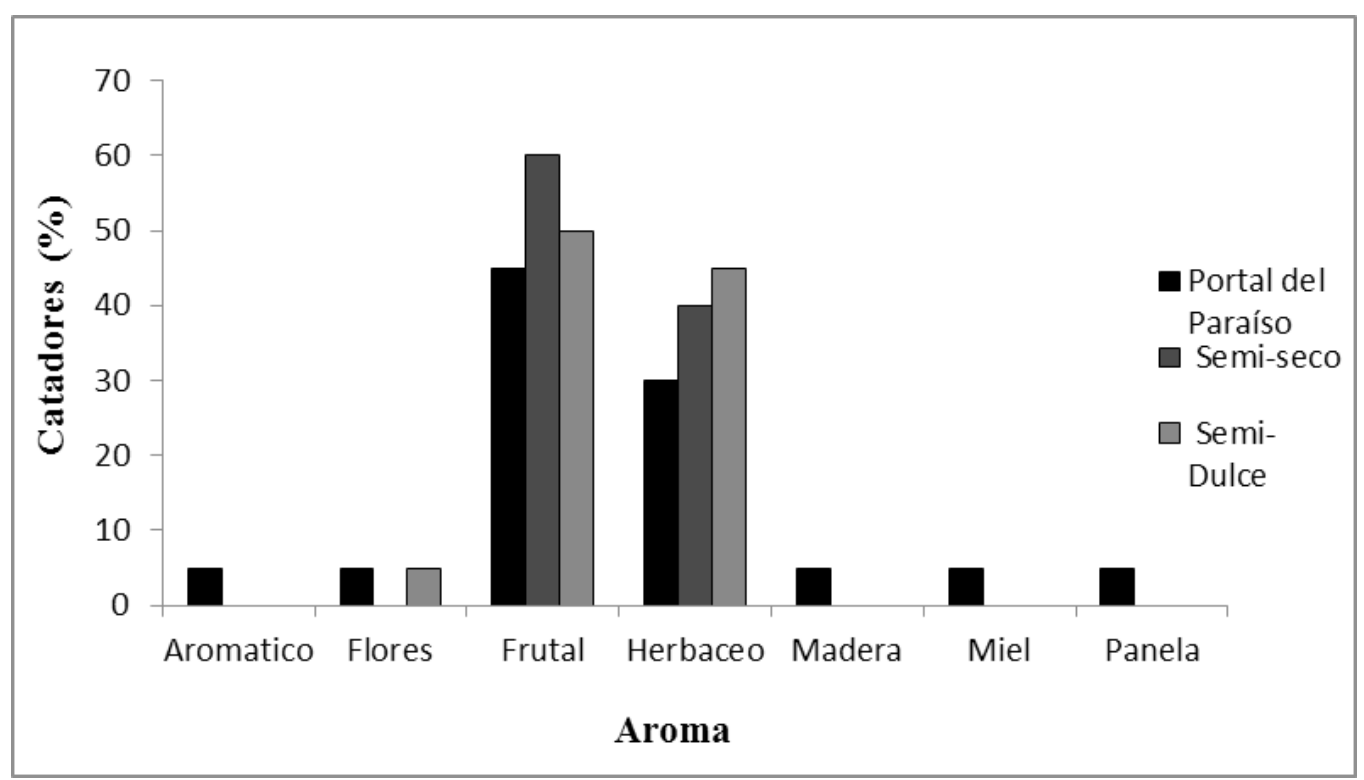

Figura 3. Percepción sensorial del aroma de los vinos Portal del Paraíso, Semiseco y Semidulce.

\section{Gusto}

Los vinos Semiseco y Semidulce presentaron una identificación de su dulzor y sequedad, ratificando su denominación con el $75 \%$ en ambos casos; lo que difiere del vino Portal del Paraíso ${ }^{\circ}$, que fue apreciado como un vino dulce por el $45 \%$ (Fig. 4). En el caso de los vinos Semiseco y Semidulce, coinciden en la caída lenta de las lágrimas, con el $55 \%$ y el $45 \%$, respectivamente. Llama la atención que el vino Portal del Paraíso presenta un $35 \%$ en la caída rápida y lenta de las lágrimas, dejando la percepción de este parámetro en forma dividida dentro del grupo de evaluadores, como se aprecia en la Fig. 5A. La existencia de lágrimas se atribuye a la molécula de glicerol (18), que tiene una cadena más larga que la del etanol, por lo que es más viscosa y tarda mayor tiempo en desprenderse de la copa que el alcohol y el agua del vino.

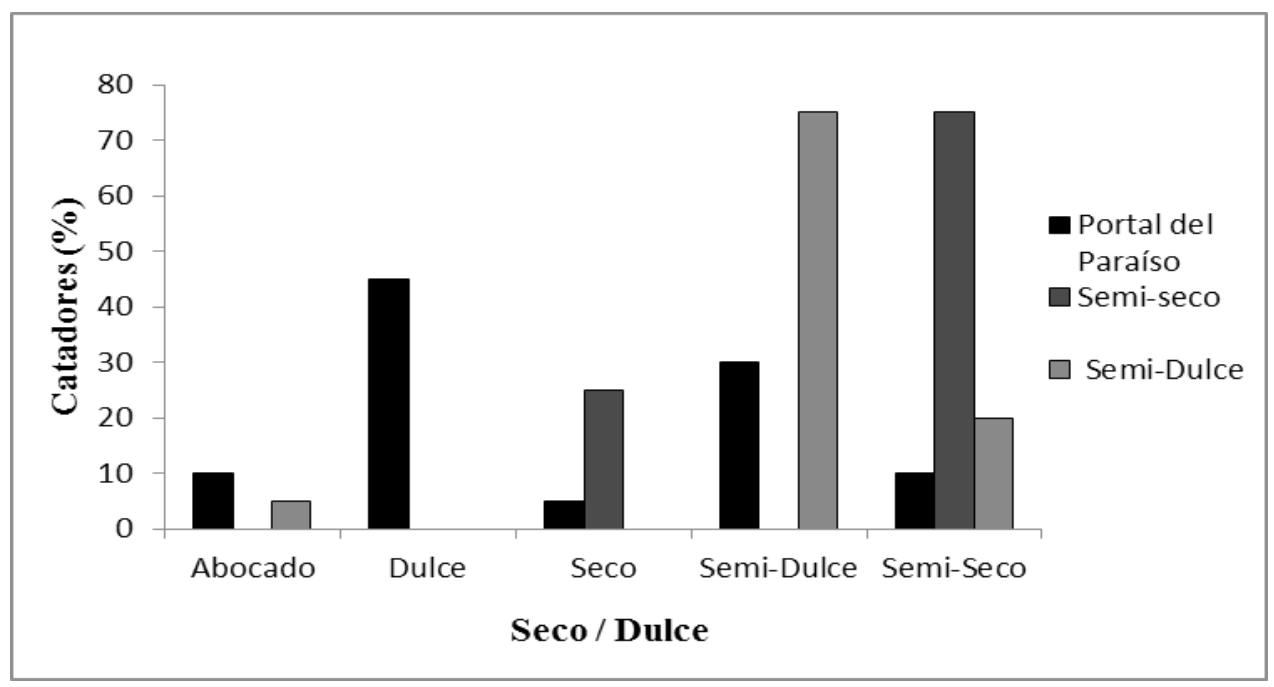

Figura 4. Percepción sensorial de la sensación seco/dulce de los vinos Portal del Paraíso, Semiseco y Semidulce. 
Todos los vinos presentaron un cuerpo medio, con porcentajes del 55\% para el Portal del Paraíso ${ }^{\oplus}$ $65 \%$ para el vino Semiseco y $60 \%$ para el vino Semidulce; con una acidez suave, según el $45 \%$ de los catadores, en el vino Portal del Paraíso ${ }^{\oplus}$, y ácida en los vinos Semiseco, con el $45 \%$, y Semidulce, con un $50 \%$ (Fig. 5 B y C).

(A)

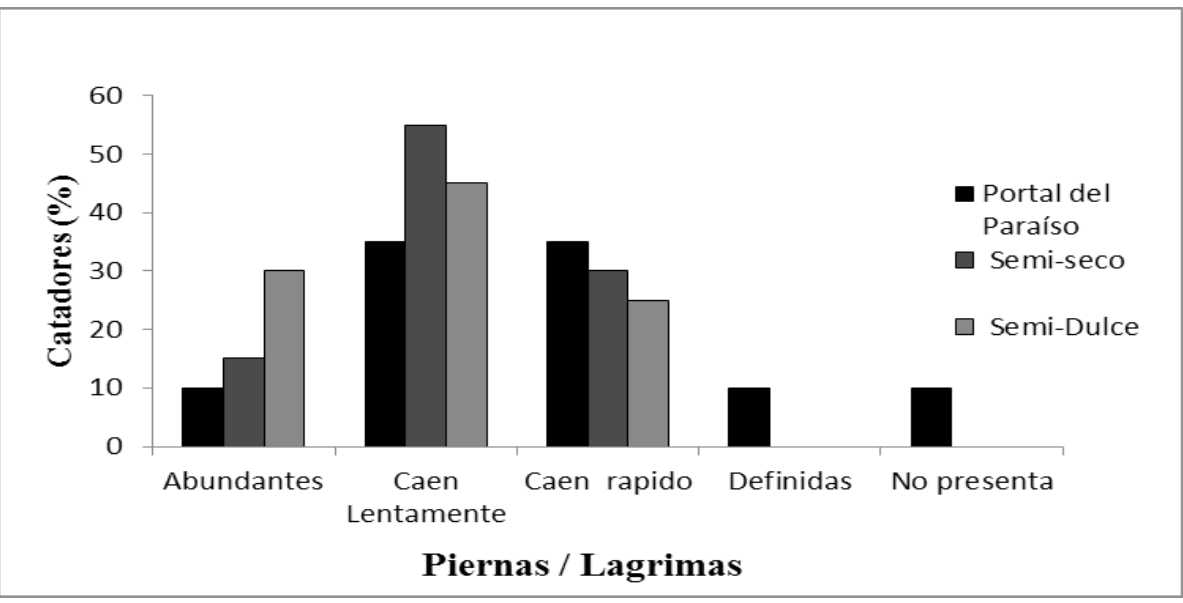

(B)

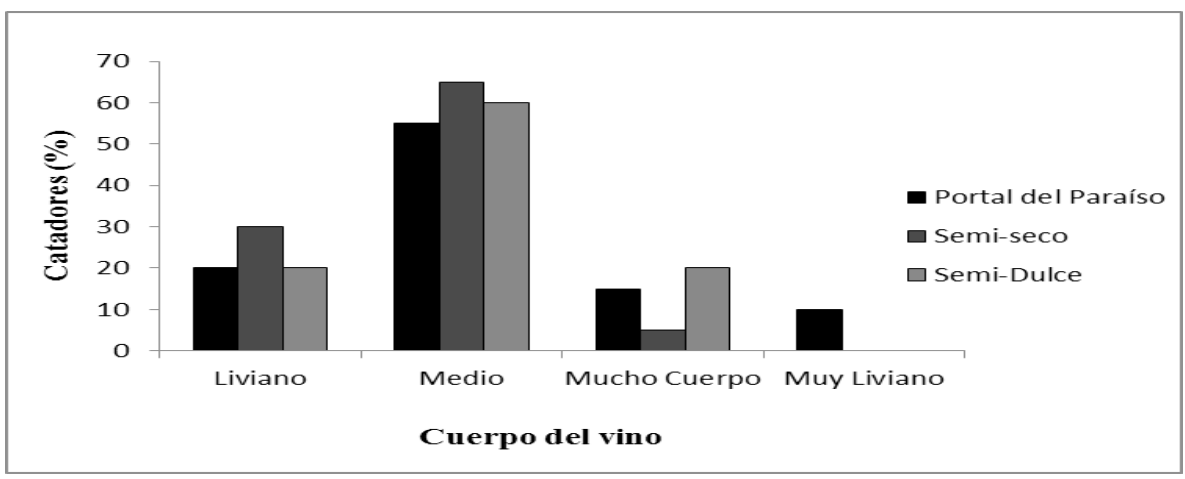

\section{(C)}

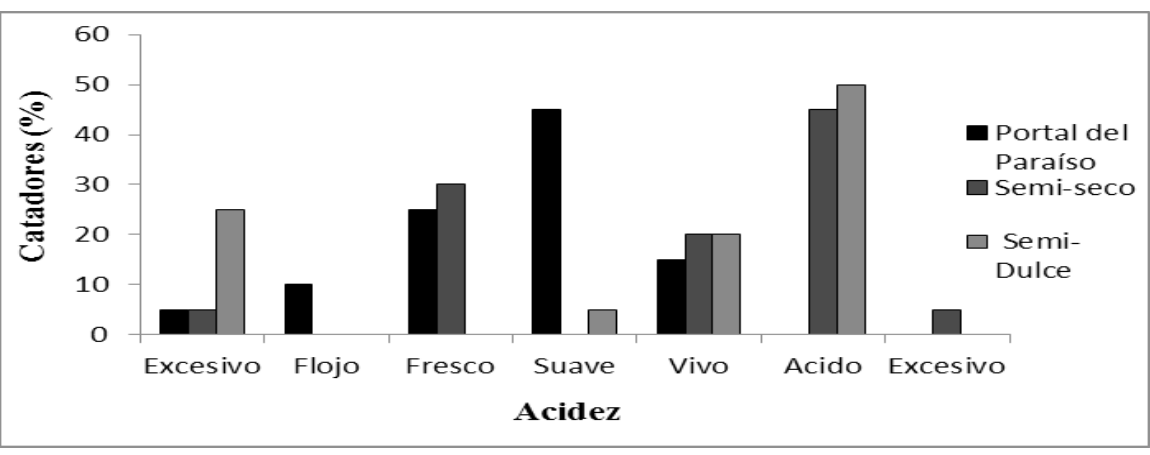

Figura 5. Percepción sensorial en A: Piernas/Lágrimas de los vinos, B: cuerpo del vino y C: Acidez de los vinos Portal del Paraíso, Semiseco y Semidulce.

En cuanto a la sensación, el vino Portal del Paraíso ${ }^{\oplus}$ es suave, de acuerdo con el $75 \%$ de los panelistas; en tanto que el Semiseco y el Semidulce son astringentes, según el $60 \%$ y el
$65 \%$, respectivamente (Fig. 6A). Una intensidad moderada, con valores del $45 \%$ para el Portal del Paraíso ${ }^{\oplus}$, el $80 \%$ para el vino Semiseco y el $60 \%$ para el Semidulce. El sabor frutal prevalece en los 
tres vinos, con el $70 \%$ para Portal del Paraíso ${ }^{\circledR}$ y el $55 \%$ para los otros dos (Fig. 6 B y C). El contenido de taninos y el método de elaboración del vino influyen en el sabor afrutado del vino, como es el caso, o, por el contrario, puede ser percibido como leñoso (8). Como resultado de lo anterior, el vino tinto se cataloga como joven, antiguo y equilibrado, de acuerdo con su sabor; los vinos jóvenes conservan el sabor frutal con cuerpo medio a bajo, con la tendencia a aumentar su gusto durante el tiempo, características similares a las encontradas en los resultados.

(A)
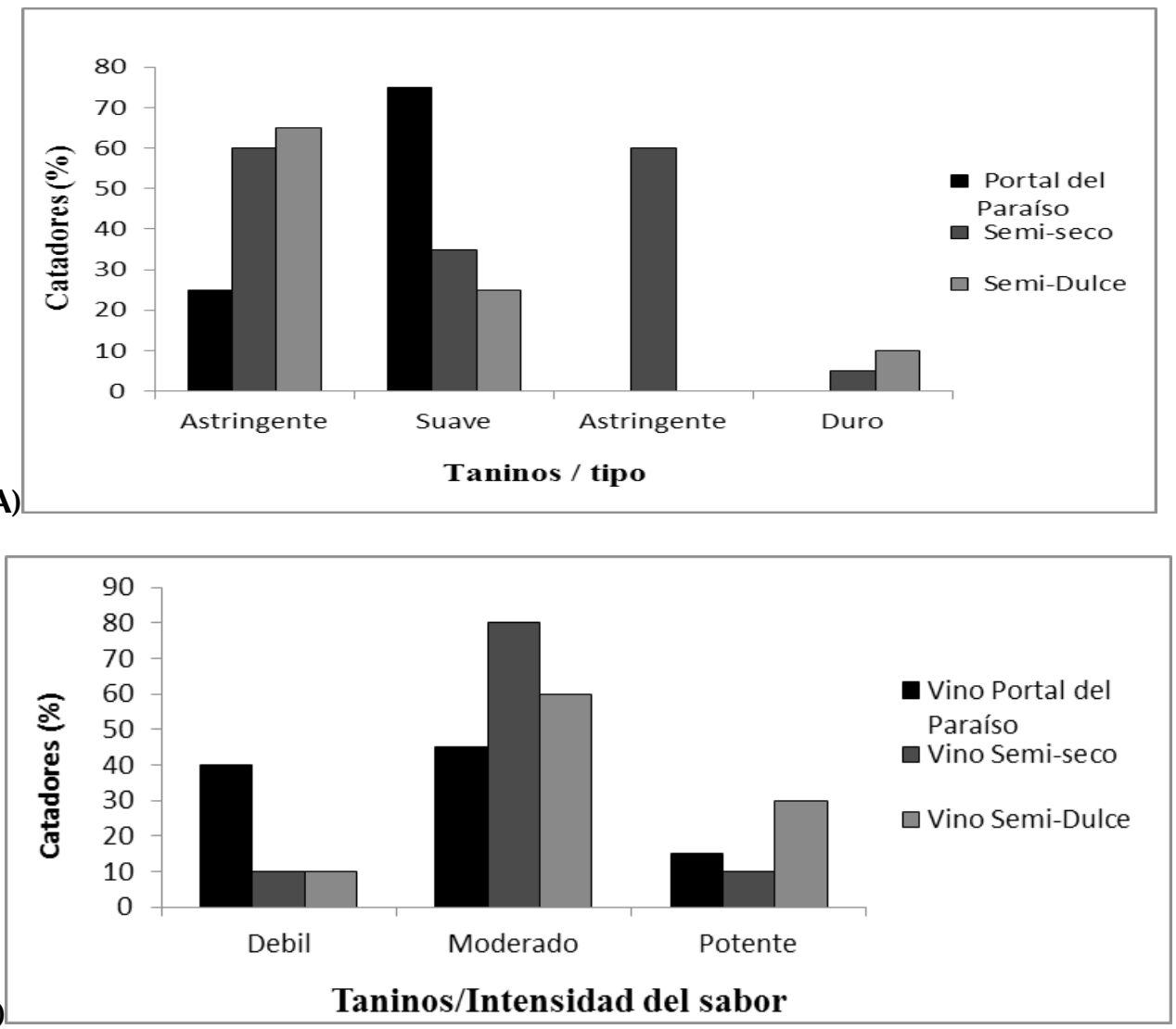

(B)

\section{Taninos/Intensidad del sabor}

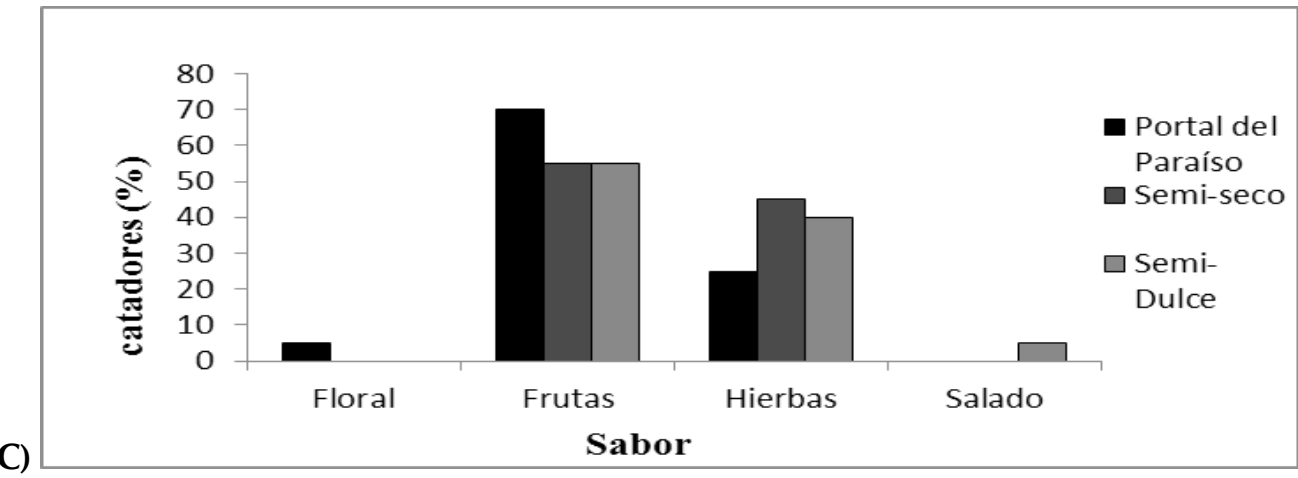

Figura 6. Percepción sensorial en A: Taninos/tipo, B: Taninos/intensidad del sabor y C: sabor de los vinos Portal del Paraíso, Semiseco y Semidulce. 


\section{Valoración general de los vinos}

Los vinos Semiseco y Semidulce fueron calificados por los panelistas como muy buenos, con puntajes de 26.6 y 37.9 , respectivamente; presentaron diferencias significativas frente al vino Portal del Paraíso ${ }^{\circledR}$, calificado como un vino correcto, con un 75.4 (Fig. 7). En todos los casos, la valoración se realizó de acuerdo con los parámetros de calificación del CINVE (8), que califica los mejores vinos con el menor puntaje; bajo este parámetro, los vinos Semiseco y Semidulce obtuvieron la calificación más baja, siendo estos, según el panel de catadores, los mejores vinos, sin presentar diferencias significativas entre ellos.

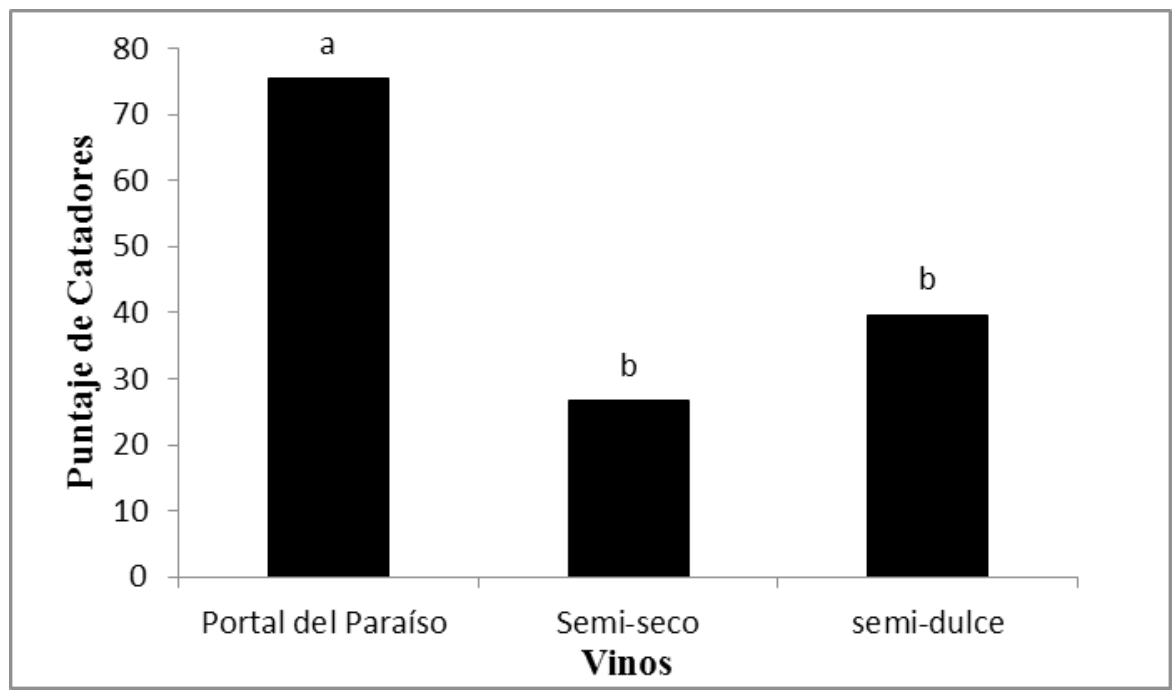

Fig. 7. Valoración de los vinos Portal del Paraíso, Semiseco y Semidulce. Promedios seguidos de letras distintas presentan diferencias estadísticas según la prueba de Tukey (5\%). El menor valor corresponde al mejor vino.

\section{Conclusiones}

Los vinos Semiseco y Semidulce tuvieron gran aceptación por los panelistas debido a sus características organolépticas; ellos los catalogaron como vinos muy buenos, por su color rubí y rojopúrpura, respectivamente, por su profundidad y limpidez, por su aroma frutal, su percepción ácida y su cuerpo medio, mostrando diferencias frente al vino Portal del Paraíso ${ }^{\oplus}$, que fue considerado un vino oscuro, color marrón, apagado, aromático y frutal, catalogándose como un vino correcto.

\section{Referencias}

(1) Almanza, P.J.; Serrano P. y Fischer, G. Manual de viticultura tropical. Tunja: UPTC, 2012.

(2) Toro, N. y Suarez, L. Obtención y caracterización del aceite de las semillas de Vitis labrusca
L. (uva Isabella) y evaluación de su actividad antioxidante. Pereira: Universidad Tecnológica de Pereira (en línea). 2012. Disponible en: http://repositorio.utp.edu.co/dspace/bitstream/11059/2943/1/66029T686.pdf.

(3) Almanza, P. Determinación del crecimiento y desarrollo del fruto de vid (Vitis viniferaL.) bajo condiciones de clima frío tropical. Tesis doctoral. Universidad Nacional de Colombia. 2011.

(4) Hernández, J.; Duran, D y Trujillo Y. Potencial fenólico de la variedad Isabella (Vitis labrusca L.) producida en Villa del Rosario-Norte de Santander-Colombia. Revista Bistua, 2010, 8(1): 88-96. Facultad de Ciencias Básicas Universidad de Pamplona. Pamplona-Colombia. 
(5) Suescún, G. Utilización vinícola de la Opuntia dillenii. Revista Bistua, 2005, 3(1): 99-106. Facultad de Ciencias Básicas Universidad de Pamplona. Pamplona-Colombia.

(6) Millan, F; G. Serrano; I. Expósito. Resveratrol. Nuestros retos en el tratamiento antienvejecimiento. Dossier. 2008.

(7) Morina, A. y Kongoll, R. Comparison of color parameters of red wines produced from albanian autochthonous grape varieties using trichromatic method. J. Agric. Sci., 2012, 11(4). 249-255. Universidad de Tirana. Albania.

(8) Peynaud, E. y Blouin, J. El gusto del vino. El gran libro de la degustación, $2^{\text {a }}$. Ed. Madrid, España. 1999. 63 p.

(9) Catania, C. y S. Avagnina. La degustación. Curso superior de degustación de vinos. EEA Mendoza. INTA. 2007a. 26 p.

(10) Guirao, G.; Cano, V.; Rodríguez, M. y Hernández, J. Evaluación del análisis sensorial de vinos de Malvasía. Universidad de la Laguna. (en línea) 2007. Disponible en: http://www. fceye.ull.es/malvasia/doc/Trabajo14.pdf.

(11) Catania, C. y Avagnina, S. El análisis sensorial. Curso superior de degustación de vinos. EEA Mendoza. INTA. 2007b. 21p.

(12) Etaio, I. Análisis sensorial del vino tinto joven de Rioja Alaversa: Descripción y evaluación de la calidad. Tesis doctorales N. ${ }^{\circ} 62$. Universidad del País Vasco. (en línea) 2009. Dispo- nible en: http://www.nasdap.ejgv.euskadi.net/ r50-public2/es/contenidos/informe_estudio/ tesis_doctorales/es_agripes/adjuntos/tesis_ doctoral62.pdf.

(13) Concurso internacional de vinos y espirituosos (CINVE). Adaptado de la hoja de cata del concurso internacional de vinos y espirituosos del año 2011 (en línea) 2011. Disponible en: http://www.cinve.es. 10 p.

(14) Î́niguez, M.; Ortega, A.; Rosales, A.; Ayala, R. y Puras, P. Estudio del color de vinos tintos de D.O.C Rioja. Logroño. 1995. 45 p.

(15) Denis, J., y M.F. Denis. Vino argentino. Manual práctico. Albatros. 2008. 47 p.

(16) Gruettner, P.; Leiva, L. y Rodillo, M. Caracterización de los racimos, evaluación del potencial productivo y enológico de diferentes cvs. de Vitis vinifera L. en las comunas de Coelemu y Trehuaco. Pontificia Universidad Católica de Chile. (en línea) 2005. Disponible en: http://www.revistavirtualpro.com/files/ TIE03_200712.pdf.

(17) Ferreira, V. La base química del aroma del vino: Un viaje analítico desde las moléculas hasta las sensaciones olfato-gustativas. Universidad de Zaragoza. Rev. Real Academia de Ciencias. 2007, 62: 7-36. Zaragoza.

(18) Phillip, H. Degustar el vino. El sabor del vino explicado. Albatros. Buenos Aires. Argentina. 2004. 191 p. 